\title{
An N-of-1 Trial of Itacitinib for a Patient with Aplastic Anemia Associated with a Gain-of-Function Variant in STAT1
}

Jacob Rosenberg ( $\sim$ JRosenberg2@mgh.harvard.edu )

Harvard Medical School

Travis Hughes

Harvard Medical School

Joshua Peters

Ragon Institute of MGH, MIT, and Harvard

Caleb Lareau

Stanford University School of Medicine https://orcid.org/0000-0003-4179-4807

Leif Ludwig

Broad Institute of MIT and Harvard https://orcid.org/0000-0002-2916-2164

\section{Lucas Massoth}

Massachusetts General Hospital

Christina Austin-Tse

Massachusetts General Hospital

\section{Heidi Rehm}

Massachusetts General Hospital

\section{Bryan Bryson}

Massachusetts Institute of Technology

\section{Sarah Fortune}

Harvard University https://orcid.org/0000-0001-7565-9975

\section{Yi-Bin Chen}

Massachusetts General Hospital

\section{Alex Shalek}

Massachusetts Institute of Technology https://orcid.org/0000-0001-5670-8778

\section{David Sykes}

Harvard University, Cambridge,

\section{Article}

Keywords: STAT1, aplastic anemia 
Posted Date: January 8th, 2021

DOI: https://doi.org/10.21203/rs.3.rs-139401/v1

License: (c) (i) This work is licensed under a Creative Commons Attribution 4.0 International License. Read Full License 


\section{An N-of-1 Trial of Itacitinib for a Patient with Aplastic Anemia Associated with a Gain-of- \\ 2 Function Variant in STAT1}

4 Jacob M. Rosenberg, ${ }^{1,2,3,6,{ }^{*}}$ Travis Hughes, ${ }^{2,3,4}$ Joshua M. Peters, ${ }^{2,5}$ Caleb A. Lareau, ${ }^{3,5}$ Leif S.

5 Ludwig, ${ }^{3,5}$ Lucas R. Massoth, ${ }^{3,10}$ Christina Austin-Tse, ${ }^{1,8,9,10}$ Heidi L. Rehm, ${ }^{1,3,5,8,10}$ Bryan Bryson, ${ }^{2,4}$

6 Sarah M. Fortune, ${ }^{2,3,6}$ Yi-Bin Chen, ${ }^{1,12}$ Alex K. Shalek, ${ }^{2,3,4,5}$ and David B. Sykes. ${ }^{1,7,11,12}$

81 Department of Medicine, Massachusetts General Hospital, Boston, MA

92 Ragon Institute of MGH, MIT, and Harvard, Cambridge, MA

103 Harvard Medical School, Boston, MA

114 Institute for Medical Engineering and Science, Department of Chemistry, and Koch Institute for Integrative Cancer

12 Research, Massachusetts Institute of Technology, Cambridge, MA

135 Broad Institute of MIT and Harvard, Cambridge, MA

146 Harvard T.H. Chan School of Public Health, Boston, MA

157 Center for Regenerative Medicine, Department of Medicine, Massachusetts General Hospital, Boston, MA

168 Center for Genomic Medicine, Massachusetts General Hospital, Boston, MA

179 Laboratory for Molecular Medicine, Partners Personalized Medicine, Cambridge, MA

1810 Department of Pathology, Massachusetts General Hospital, Boston, MA

1911 Harvard Stem Cell Institute, Cambridge, MA

2012 Massachusetts General Hospital Cancer Center, Boston, MA

* Contact information:

23 Jacob M. Rosenberg

2455 Fruit Street

25 Boston, MA, 02114, USA

26 JRosenberg2@mgh.harvard.edu

$27 \quad 860-930-5744$ 
Abstract

30

An 18-year-old man presented with aplastic anemia, and exome sequencing identified a germline gain-of-function variant in the gene STAT1. Treatment with itacitinib, an investigational selective Janus Kinase 1 (JAK1) inhibitor, resulted in prompt recovery of hematopoiesis. An exhausted CD8+ T cell population and myeloid populations enriched for an interferon- $\gamma$ signature correlated with disease activity. Patient bone marrow sections displayed increased phospho-STAT1 staining, as did other idiopathic aplastic anemia cases, suggesting a shared pathophysiologic mechanism. This study describes the success and mechanism of a molecularly targeted therapy with potential implications for the treatment of aplastic anemias and other autoimmune disorders.

\section{Introduction}

An 18-year-old man presented with 3 weeks of pallor, weakness, and dyspnea. On exam, the patient was pale but well appearing with a BMI of 17 . He had buccal aphthous ulcers and thrush on the soft palate. His peripheral blood laboratory values were notable for pancytopenia with a hemoglobin level of $3.9 \mathrm{~g} / \mathrm{dL}$ (reference range 12-16) without signs of hemolysis, a white blood cell count nadir of 350 cells $/ \mu$ l (reference range 4,500-11,000), an absolute neutrophil count nadir of $210 \mathrm{cells} / \mu \mathrm{l}$ (reference range $1,800-7,700)$, and a platelet count of $118,000 / \mu$ l (reference range $140,000-430,000)$. He was transfused red blood cells, and a bone marrow biopsy demonstrated hypocellularity (20-30\% cellularity) with a myeloid predominance, absence of erythroid precursors, and decreased megakaryocytes. An infectious and rheumatologic workup was unrevealing (Fig. S1). Testing for paroxysmal nocturnal hemoglobinuria, Fanconi anemia, and telomere shortening were negative. Bone marrow panel mutational testing, karyotypic analysis, and T-cell receptor spectrotyping were all within normal limits. Though platelets were relatively preserved, based on criteria of peripheral neutrophils $<500 / \mathrm{mL}$, reticulocytes $<1 \%$, and bone marrow hypocellularity, a diagnosis of severe aplastic anemia was made. ${ }^{1}$ 
53 His past medical history was notable for recurrent severe oral aphthous ulcers since childhood, for

54 which he took prednisone $10 \mathrm{mg}$ during flares. Given his thrush, we prescribed nystatin oral

55 suspension which he took as needed. His paternal grandmother, father, and other family members

56 suffered similar oral ulcers since youth, and construction of a pedigree suggested an autosomal

57 dominant pattern of inheritance (Fig. 1A). His father also suffered from thrush, but additional family

58 history beyond these clinical features was unable to be obtained. No other family members were

59 known to have aplastic anemia.

\section{Results}

$61 \quad$ Molecular Evaluation

62 Aplastic anemia is a rare disorder characterized by pancytopenia due to bone marrow failure. It can

63 be caused by direct damage (e.g. toxins or radiation), rare genetic syndromes, infection, or, most

64 commonly, idiopathic autoimmune T cell attack of hematopoietic cells. ${ }^{2}$ Standard-of-care therapy

65 includes allogeneic stem cell transplantation or intensive immunosuppression.

66 Given the autosomal dominant inheritance pattern of the patient's aphthous lesions and unclear

67 etiology of his aplastic anemia, we performed exome sequencing of both patient and father. A

68 heterozygous variant (c.800C>T, p.Ala267Val) in the coiled-coil domain of STAT1 was identified in

69 both the patient and his father and confirmed by Sanger sequencing (Fig. 1B).

70 STAT1 is a transcription factor downstream of JAK signaling, a pathway critical to hematopoiesis,

71 immunity, and development. ${ }^{3}$ More than 50 cytokines and growth factors, including interferons, signal

72 through this pathway by binding to cognate receptors and effecting dimerization. Dimerization

73 activates receptor-bound JAKs which then phosphorylate and activate STATs. STATs form either

74 homo- or hetero-dimers, translocate into the nucleus, bind specific DNA sequences, and activate

75 discrete transcriptional programs.

76 Gain of function (GOF) variants in STAT1 cause an autosomal dominant syndrome with a spectrum of

77 autoimmune features and near complete penetrance of mucocutaneous candidiasis. ${ }^{4,5}$ More than 400 
78 patients with more than 100 different STAT1 GOF variants have been reported. ${ }^{4,6,7}$ Aplastic anemia

79 has been described, as have numerous other autoimmune cytopenias, and severe aphthous

80 stomatitis is frequent. ${ }^{4,6,8,9}$ GOF variants lead to hyper-phosphorylation of STAT1 in response to

81 stimulation, increasing STAT1-dependent transcription..$^{7,10,11}$ The p.Ala267Val variant identified in our

82 patient is absent from large population databases, but has been identified in more than 10 individuals

83 in multiple families with chronic mucocutaneous candidiasis, and shown to have a GOF effect. ${ }^{6,12}$ We

84 thus considered the p.Ala267Val variant as causal for our patient's recurrent aphthous ulcers, thrush,

85 and aplastic anemia.

\section{$86 \quad$ Itacitinib Trial}

87 As STAT1 activation is heavily dependent on JAK signaling, the JAK inhibitors ruxolitinib and

88 tofacitinib have been trialed in STAT1 GOF patients. Successful and unsuccessful cases have been

89 reported for the treatment of alopecia, diabetes, thrush, fungal infections, and autoimmune

90 cytopenias. ${ }^{8,13-18}$ Ruxolitinib and tofacitinib inhibit both JAK1 and JAK2 with the potential for anemia

91 and thrombocytopenia, since JAK2 is downstream of the erythropoietin and thrombopoietin receptors.

92 In considering a JAK inhibitor, we thus sought to avoid JAK2 inhibition.

93 The investigational drug itacitinib is a potent JAK1 inhibitor with an $\mathrm{IC}_{50}$ of $3.2 \mathrm{nM}$ and selectivity for

94 JAK1. ${ }^{19}$ Itacitinib has been studied in myeloproliferative neoplasms, graft-versus-host disease, and

95 autoimmune disorders.

96 The patient remained dependent on red blood cell transfusions, and he was thrice admitted to the

97 hospital for febrile neutropenia. Preparations were underway for hematopoietic stem cell

98 transplantation when, based on genotyping results, we enrolled the patient in a single patient expanded use trial of itacitinib (https://clinicaltrials.gov/ct2/show/NCT03906318). On day 169 after presentation, he received his weekly transfusion and initiated itacitinib $300 \mathrm{mg}$ daily. The following week, and for all subsequent visits after the initiation of itacitinib, laboratory values demonstrated resolution of neutropenia and anemia, and the patient became red blood cell transfusion independent

103 (Fig. 1C). A subsequent bone marrow biopsy demonstrated a return of trilineage hematopoiesis. 
Rosenberg et al.

104 Increased STAT1 signaling as measured by phospho-STAT1 (pSTAT1) staining, which had been

105 present at time of diagnosis, resolved after treatment (Fig. 1D). The patient's thrush and oral ulcers

106 persisted but at significantly decreased frequency and severity. Clinically, fatigue and weight loss

107 resolved, and he returned to schooling. He completed 20 months of itacitinib therapy without any

108 adverse events before electing to self-discontinue. Aplastic anemia has not recurred.

109 Immunological Profiling

110 To understand the cellular mechanisms involved in the pathogenesis and resolution of this patient's

111 aplastic anemia, we performed comprehensive immunophenotyping. To confirm STAT1 GOF activity,

112 we used phospho-CyTOF to measure phospho-STAT1 in monocytes after stimulation with interferon- $\gamma$

113 at timepoints pre- and post-itacitinib treatment. At all timepoints, the patient's monocytes had higher

114 levels of pSTAT1 than healthy control. Post-itacitinib, pSTAT1 levels decreased, though not to the

115 level of healthy control (Fig. 2A).

116 The largest studies of STAT1 GOF patients have demonstrated increased frequencies of $T_{H} 1$ and

117 decreased frequencies of $T_{H} 17$ T cells. ${ }^{20,21}$ Increased signaling through STAT1 biases T cell

118 differentiation into the $T_{H} 1$ phenotype, which is characterized by secretion of interferon- $\gamma$. $T_{H} 1$

119 differentiation comes at the expense of STAT3-mediated $\mathrm{T}_{\mathrm{H}} 17$ differentiation, which is characterized

120 by secretion of IL-17A and cytokines critical for immunity to mucocutaneous Candida. It is

121 hypothesized that JAK inhibition may restore the balance between $T_{H} 1$ and $T_{H} 17$ differentiation by

122 dampening STAT1 signaling. ${ }^{8}$

123 Interrogating the $T_{H} 1$ and $T_{H} 17$ pathways, we compared plasma cytokine levels pre- and post-

124 treatment with itacitinib. This identified significant decreases in the $T_{H} 1$ cytokines interferon- $\gamma$ and

125 IL-12p40 following therapy, but no significant shifts in the $T_{H} 17$ cytokines IL-17A or IL-17F (Fig. 2B).

126 Of note, elevated serum interferon- $\gamma$ has been described in aplastic anemia. ${ }^{22}$

127 Examining CD4+ T cells by post-stimulation intracellular cytokine staining, we found a near absence

128 of IL-17A-producing CD4+ T cells $\left(T_{H} 17\right)$ in the patient compared to healthy controls; this did not 
129 change with treatment (Fig. S2B). Frequencies of interferon- $\gamma$-producing CD4+ T cells $\left(T_{H} 1\right)$ did not

130 differ between healthy controls, the patient pre-itacitinib, or the patient post-itacitinib (Fig. 2C). Thus,

131 we did not observe evidence of itacitinib restoring a balance between STAT1-mediated $T_{H} 1$ and

132 STAT3-mediated $T_{H} 17$ frequencies. Moreover, while we observed other peripheral blood mononuclear

133 cells (PBMC) abnormalities including an absence of $\mathrm{T}_{\text {reg }}$ cells, elevated frequencies of memory CD4+

134 and CD8+ T cells, and reduced frequencies of $T$ follicular helper $\left(T_{F H}\right)$ cells compared to healthy

135 controls (Fig. S2A), none of these frequencies changed significantly with itacitinib treatment.

136 Surprisingly, the patient's pre-itacitinib CD8+ T cells produced significantly less interferon- $\gamma$ than

137 healthy controls in vitro (Fig. 2D). After treatment with itacitinib, CD8+ interferon- $\gamma$ production

138 increased but not to the level of healthy controls. Pre-itacitinib memory CD8+ T cells also had a

139 striking increase in expression of the activation marker Programmed Death 1 (PD-1), which

140 decreased with itacitinib treatment (Fig. 2E). Thus PD-1+ CD8+ T cells-which are defective in their

141 ability to secrete interferon- $\gamma$ upon stimulation in vitro-closely correlate with aplastic anemia disease

142 activity.

143 Taken together, these findings suggest a model whereby during aplastic anemia, the patient's CD8+

144 T cells are chronically activated against hematopoietic progenitors in vivo leading to an exhausted

145 phenotype characterized by impaired ability to secrete interferon- $\gamma$ in vitro and increased PD-1

146 expression. By inhibiting JAK1-driven activation, itacitinib relieved CD8+ T cell exhaustion. Consistent

147 with our findings, elevated frequencies of activated CD8+ T cells have been described in aplastic

148 anemia. ${ }^{23}$ We did observe the seemingly paradoxical finding of increased serum interferon- $\gamma$ (Fig. 2B)

149 yet decreased interferon- $\gamma$ secretion by CD8+ T cells in vitro (Fig. 2D). One interpretation of these

150 results is that these CD8+ T cells are chronically secreting interferon-gamma in vivo, yet their

151 exhausted phenotype reduces their capacity to secrete interferon-gamma in vitro upon stimulation. ${ }^{24}$

152 To explore the in vivo unstimulated cytokine effector function of these cells, we performed single-cell

153 transcriptional analyses. 
154 Single-Cell Gene Expression Analysis

155 To understand the transcriptional programs governing our patient's aplastic anemia, we performed 156 single-cell transcriptional sequencing (scRNA-Seq), comparing PBMCs from healthy controls and our 157 patient at timepoints before and after itacitinib. Unsupervised clustering demonstrated that healthy, 158 pre-itacitinib, and post-itacitinib cells represent distinct clusters. Post-itacitinib samples clustered 159 between healthy and pre-itacitinib samples, suggesting progression from disease towards the healthy 160 state (Fig. 3A).

161 We then separately subclustered T cell-containing and myeloid populations for further analysis (Fig.

162 S3). T cell-containing cells from the patient and healthy controls subclustered into a CD8+ population, 163 NK population, and mixed CD4+ and CD8+ memory and naïve populations. Given the activated but 164 dysfunctional state of our patient's CD8+ T cells, we scored all CD8+ T cells for cytotoxicity, cytokine 165 effector function, and exhaustion (Table S1). Each of these scores were elevated in the patient's pre166 itacitinib cells compared to healthy controls, confirming their cytotoxic and exhausted phenotype (Fig. 167 3B). After treatment, exhaustion and cytokine effector scores decreased. Our finding of increased 168 transcriptional cytokine effector scores in the patient's pre-itacitinib CD8+ T cells supports the 169 hypothesis that these cells secrete higher levels of cytokines such as interferon- $\gamma$ in vivo, despite their reduced capacity to secrete interferon- $\gamma$ in vitro upon stimulation.

171 Subclustering of myeloid populations identified five populations (Fig. 3C). Of note, "activated CD14+ 172 monocytes" and "C1Q+ monocytes" were found virtually exclusively in the patient's and not in the 173 healthy controls' cells. Both of these subclusters expressed STAT1 activation-induced genes such as 174 the complement genes $C 1 Q C, C 1 Q B, C 1 Q A$ or the interferon-inducible genes FAM26F or GBP1, 175 respectively (Fig. S3). Type I interferons ( $\alpha, \beta$, and others) and Type II interferon (only $\gamma$ ) induce 176 overlapping but distinct transcriptional signatures. Since monocytes express high levels of the 177 interferon- $\gamma$ receptor, we scored each myeloid cell to assess for interferon exposure (Table S1). Both 178 Type I and II interferon scores in pre-treatment monocytes were higher than healthy control or post- 
179 itacitinib monocytes. Within pre-itacitinib monocytes, Type II scores were higher than Type I,

180 suggesting a primarily interferon- $\gamma$ induced state $\left(p<10^{-10}\right.$, Wilcoxon rank-sum).

181 Single-Cell Epigenetic Analysis

182 To understand the epigenetic effects of the STAT1 GOF variant and itacitinib treatment, we performed

183 single-cell Assay for Transposase-Accessible Chromatin with Sequencing (scATAC-seq). Within

184 effector CD8+ T cells, we saw increased accessibility at the PD-1 locus, consistent with our

185 observation of increased PD-1 protein expression (Fig. 2E), and this accessibility decreased after

186 treatment (Fig. 3D and S4).

187 Genome-wide, STAT1 motif accessibility pre-itacitinib was increased when compared to healthy

188 controls or post-itacitinib samples (Fig. 3E). These studies suggest that changes in accessible

189 chromatin correlate with STAT1-mediated autoimmunity and can be reversed with itacitinib.

190 pSTAT1 Bone Marrow Immunostaining

191 Immunostaining our patient's bone marrow sections, we found increased pSTAT1 staining pre-

192 itacitinib, and this STAT1 activity resolved following itacitinib therapy (Fig. 1D). To determine whether

193 similar STAT1 dysregulation exists in patients with idiopathic (non-STAT1-mutated) aplastic anemia, 194 we analyzed bone marrow sections from other aplastic anemia patients. Compared to healthy donor 195 marrow in which no staining was detected, three of four marrows exhibited positive pSTAT1 staining

196 (Fig. 4). This raises the exciting possibility that STAT1 activation is a feature of a subset of aplastic 197 anemias for which pSTAT1 is a potential biomarker.

\section{Discussion}

Here we present a man with a history of oral ulcers inherited in an autosomal dominant pattern who 200 developed aplastic anemia. Exome sequencing identified a pathogenic GOF mutation in STAT1. 201 Molecular inhibition of JAK1, a kinase upstream of STAT1 activation, resulted in resolution of the 202 patient's aplastic anemia. 
203 Longitudinal immunophenotyping of samples unperturbed by confounding immunosuppressive 204 therapy revealed an expanded, activated, cytolytic, and exhausted memory CD8+ T cell population 205 that correlated with disease activity, as did plasma levels of interferon- $\gamma$. After itacitinib treatment, 206 these abnormalities improved, with parallels to mouse and human models of idiopathic aplastic 207 anemia. ${ }^{23,25}$

208 To our knowledge, this represents the first report of a targeted therapy for the treatment of 209 autoimmune aplastic anemia. This case also marks the first report of itacitinib for use in a primary 210 immunodeficiency. Given the high frequency of cytopenias in primary immunodeficiencies, itacitinib's 211 JAK1 selectivity may be of particular clinical benefit. ${ }^{26,27}$

212 Establishing the causality of a therapy in a single-patient trial is challenging, but the co-incident timing 213 of itacitinib with near immediate hematopoietic recovery after 6 months of transfusion-dependence, as 214 well as the low expected rate of spontaneous recovery in aplastic anemia, ${ }^{2}$ are evidence in favor of a 215 therapeutic effect of itacitinib in this case.

216 While STAT1 GOF is a rare condition, aplastic anemia is more common. Aplastic anemia can be 217 triggered by a spectrum of host and environmental factors, ${ }^{2}$ but our patient's immunophenotypic 218 similarities to other cases of aplastic anemia raise the possibility of a shared downstream 219 pathophysiology for which JAK inhibitor therapy could be effective. Additionally, our finding of 220 increased bone marrow pSTAT1 staining in a majority of aplastic anemia cases may be a useful 221 biomarker to identify candidate patients. We hope these results spur larger trials to answer these 222 questions, particularly given the relative tolerability of JAK inhibitors compared to current standard 223 treatment modalities including hematopoietic stem cell transplantation or medical 224 immunosuppression. 
Rosenberg et al.

225 Online Methods

226 Please see separate attachment for methods section.

227

228

Page 10 


\section{Acknowledgements}

230 Funding was provided by the Massachusetts General Hospital Department of Medicine Pathways 231 program and NIH T32 AI007387 through the Divisions of Infectious Diseases at MGH and Brigham 232 and Women's Hospital. We thank Katrina Armstrong, Mark Fishman, Victor Fedorov, Lauren Zeitels, 233 Rajesh Ranganathan, and Alex Soltoff for establishing and guiding the MGH Pathways Program, Mike 234 Waring, Maris Handley, Patricia Grace, Fred Preffer, and David Dombkowski for assistance with flow 235 cytometry, Robert Hasserjian for historical aplastic anemia cases, and Candice Del Rio for clinical 236 research assistance. We thank the Stanford Human Immune Monitoring Core for assistance in 237 measuring plasma cytokine levels and phospho-CyTOF analysis.

\section{Author Contributions}

239 J.M.R. conceived the study and designed the experiments with D.B.S. J.M.R., T.H., J.M.P., C.A.L., 240 L.S.L., and L.R.M. performed the experiments and analyzed the data. C.A.T. and H.L.R performed the 241 exome sequencing. B.B., A.K.S., Y.B.C., S.M.F., and D.B.S supported the studies and reviewed the 242 manuscript.

\section{Competing Interests}

244 Y.B.C. reports consulting fees from Incyte. All other authors declare no competing interests. 


\section{References}

248 1. Camitta, B. M., Storb, R. \& Thomas, E. D. Aplastic Anemia. N. Engl. J. Med. 306, 712-718 (1982).

249 2. Young, N. S. Aplastic Anemia. N. Engl. J. Med. 379, 1643-1656 (2018).

250 3. Morris, R., Kershaw, N. J. \& Babon, J. J. The molecular details of cytokine signaling via the 251 JAK/STAT pathway. Protein Sci. Publ. Protein Soc. 27, 1984-2009 (2018).

252 4. Toubiana, J. et al. Heterozygous STAT1 gain-of-function mutations underlie an unexpectedly 253 broad clinical phenotype. Blood 127, 3154-3164 (2016).

254 5. van de Veerdonk, F. L. et al. STAT1 Mutations in Autosomal Dominant Chronic Mucocutaneous 255 Candidiasis. N. Engl. J. Med. 365, 54-61 (2011).

256 6. Depner, M. et al. The Extended Clinical Phenotype of 26 Patients with Chronic Mucocutaneous 257 Candidiasis due to Gain-of-Function Mutations in STAT1. J. Clin. Immunol. 36, 73-84 (2016).

258 7. Okada, S. et al. Human STAT1 Gain-of-Function Heterozygous Mutations: Chronic 259 Mucocutaneous Candidiasis and Type I Interferonopathy. J. Clin. Immunol. 40, 1065-1081 260 (2020).

261 8. Weinacht, K. G. et al. Ruxolitinib reverses dysregulated T helper cell responses and controls 262 autoimmunity caused by a novel signal transducer and activator of transcription 1 (STAT1) gain263 of-function mutation. J. Allergy Clin. Immunol. 139, 1629-1640.e2 (2017).

264 9. Leiding, J. W. et al. Hematopoietic stem cell transplantation in patients with gain-of-function signal 265 transducer and activator of transcription 1 mutations. J. Allergy Clin. Immunol. 141, 704-717.e5 266 (2018).

267 10. Fujiki, R. et al. Molecular mechanism and structural basis of gain-of-function of STAT1 caused by 268 pathogenic R274Q mutation. J. Biol. Chem. 292, 6240-6254 (2017).

269 11. Kagawa, R. et al. Alanine-scanning mutagenesis of human STAT1 to estimate loss- or gain-of270 function variants. J. Allergy Clin. Immunol. 140, 232-241 (2017).

271 12. Zimmerman, O. et al. STAT1 Gain-of-Function Mutations Cause High Total STAT1 Levels With $272 \quad$ Normal Dephosphorylation. Front. Immunol. 10, 1433 (2019). 
Rosenberg et al.

STAT1 GOF in Aplastic Anemia

273 13. Bloomfield, M. et al. Utility of Ruxolitinib in a Child with Chronic Mucocutaneous Candidiasis

274 Caused by a Novel STAT1 Gain-of-Function Mutation. J. Clin. Immunol. 38, 589-601 (2018).

275

276

277

14. Forbes, L. R. et al. Jakinibs for the treatment of immune dysregulation in patients with gain-of-

15. Higgins, E. et al. Use of ruxolitinib to successfully treat chronic mucocutaneous candidiasis caused by gain-of-function signal transducer and activator of transcription 1 (STAT1) mutation. J. Allergy Clin. Immunol. 135, 551-553.e3 (2015).

16. Mössner, R. et al. Ruxolitinib Induces Interleukin 17 and Ameliorates Chronic Mucocutaneous Candidiasis Caused by STAT1 Gain-of-Function Mutation. Clin. Infect. Dis. 62, 951-953 (2016).

17. Chaimowitz, N. S., Ebenezer, S. J., Hanson, I. C., Anderson, M. \& Forbes, L. R. STAT1 Gain of Function, Type 1 Diabetes, and Reversal with JAK Inhibition. N. Engl. J. Med. 383, 1494-1496 (2020).

18. Zimmerman, O. et al. Risks of Ruxolitinib in STAT1 Gain-of-Function-Associated Severe Fungal Disease. Open Forum Infect. Dis. 4, ofx202 (2017).

19. Kettle, J. G. et al. Inhibitors of JAK-family kinases: an update on the patent literature 2013-2015, part 1. Expert Opin. Ther. Pat. 27, 127-143 (2017).

20. Liu, L. et al. Gain-of-function human STAT1 mutations impair IL-17 immunity and underlie chronic mucocutaneous candidiasis. J. Exp. Med. 208, 1635-1648 (2011).

21. Zheng, J. et al. Gain-of-function STAT1 mutations impair STAT3 activity in patients with chronic mucocutaneous candidiasis (CMC). Eur. J. Immunol. 45, 2834-2846 (2015).

22. Zoumbos, N. C., Gascon, P., Djeu, J. Y. \& Young, N. S. Interferon is a mediator of hematopoietic suppression in aplastic anemia in vitro and possibly in vivo. Proc. Natl. Acad. Sci. 82, 188-192 (1985).

23. Hosokawa, K. et al. Memory Stem T Cells in Autoimmune Disease: High Frequency of Circulating CD8+ Memory Stem Cells in Acquired Aplastic Anemia. J. Immunol. 196, 1568-1578 (2016). 
299 24. McLane, L. M., Abdel-Hakeem, M. S. \& Wherry, E. J. CD8 T Cell Exhaustion During Chronic Viral 300 Infection and Cancer. Annu. Rev. Immunol. 37, 457-495 (2019).

301 25. Smith, J. N. P., Kanwar, V. S. \& MacNamara, K. C. Hematopoietic Stem Cell Regulation by Type I 302 and II Interferons in the Pathogenesis of Acquired Aplastic Anemia. Front. Immunol. 7, 330 303 (2016).

304 26. Gadina, M. et al. Janus kinases to jakinibs: from basic insights to clinical practice. Rheumatol. 305 Oxf. Engl. 58, i4-i16 (2019).

306 27. Sanchez, G. A. M. et al. JAK1/2 inhibition with baricitinib in the treatment of autoinflammatory 307 interferonopathies. J. Clin. Invest. 128, 3041-3052 (2018). 
a.

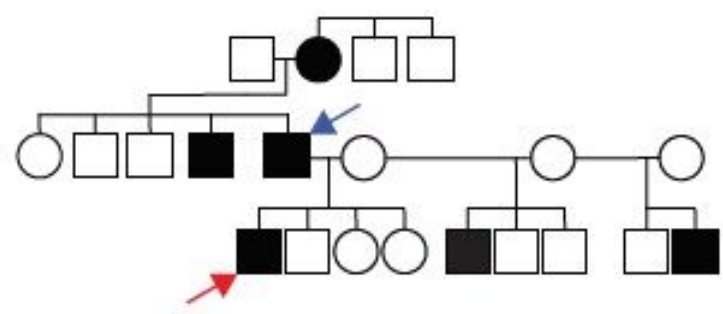

c.
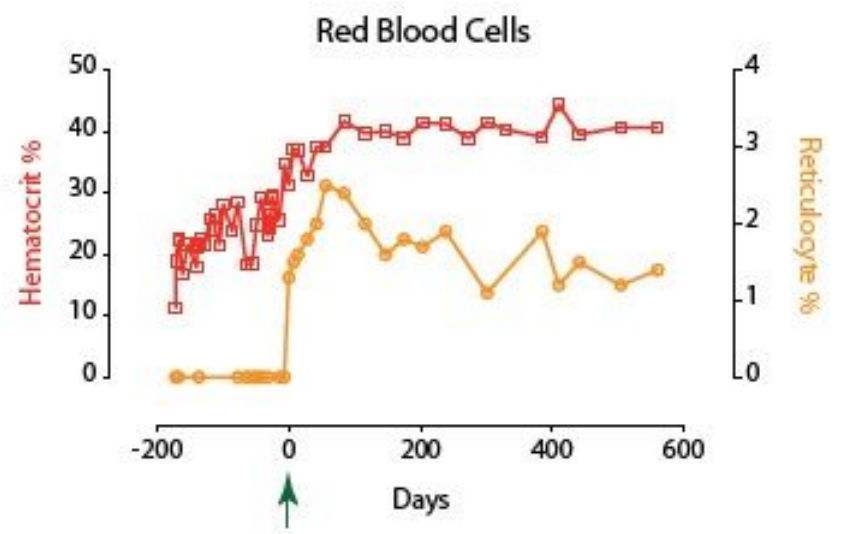

d.

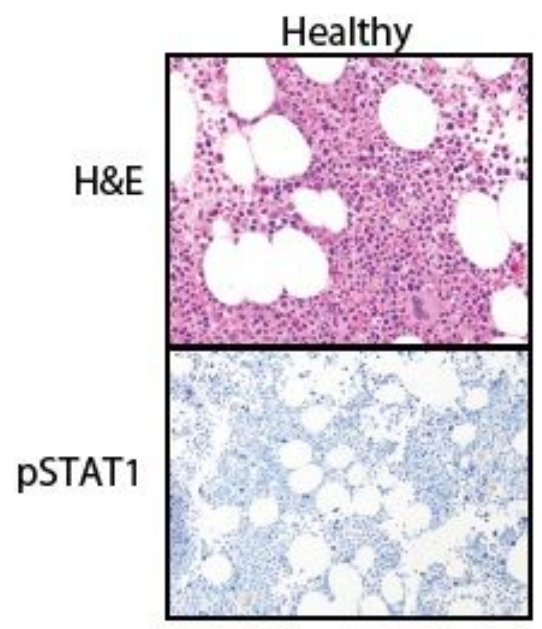

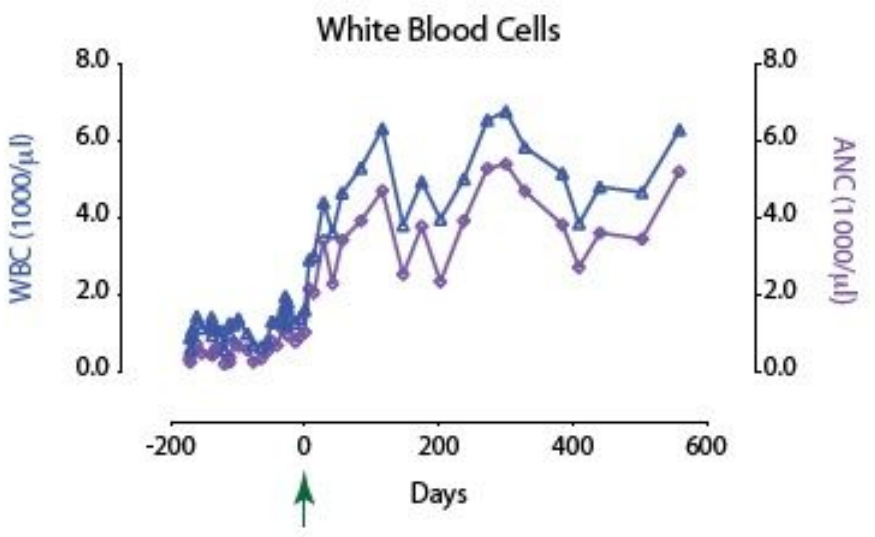

b.

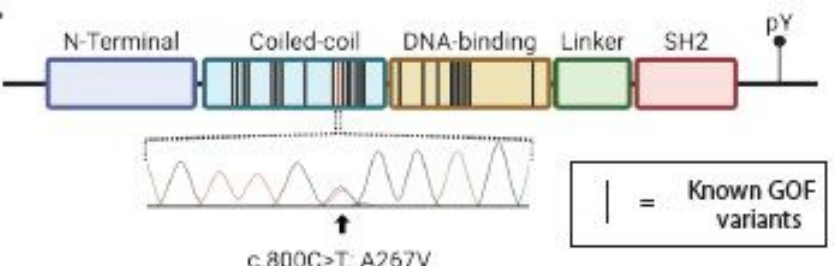

$800 \mathrm{C} \rightarrow \mathrm{T} ; \mathrm{A} 267 \mathrm{~V}$

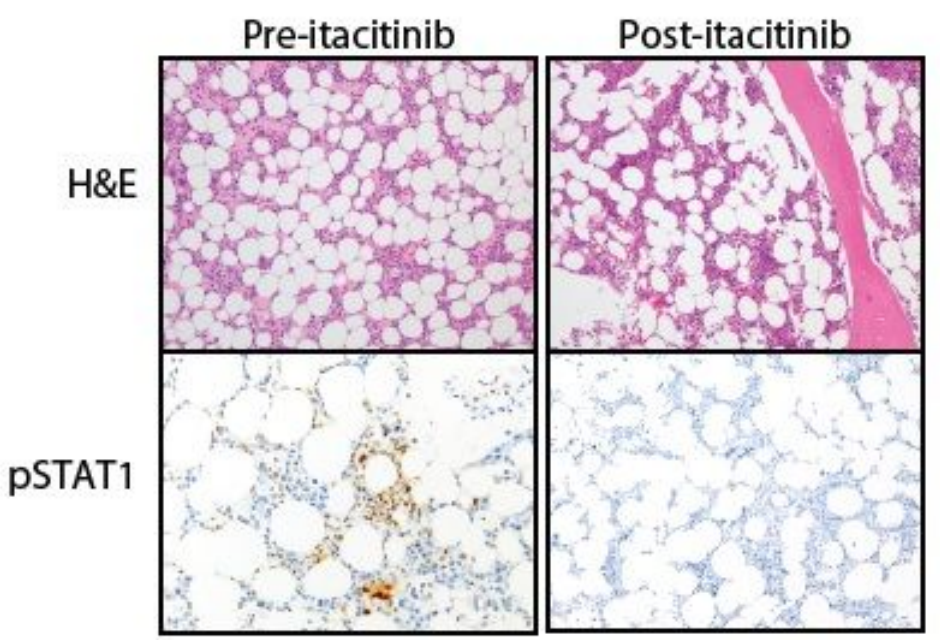

\section{Figure 1}

Clinical presentation and response to itacitinib. a. Family pedigree. Males and females are denoted by squares and circles, respectively. The phenotype of severe oral ulcers is demonstrated by shaded symbols. The patient and his father, both heterozygous for the A267V mutation, are noted by the red and blue arrows, respectively. b. Schematic of the domains of the STAT1 protein and its tyrosine phosphorylation site (pY). Black bars represent known STAT1 GOF mutations, the red bar represents the patient's mutation, and Sanger sequencing of the mutation is shown. c. Peripheral blood laboratory 
values and response to itacitinib. Hematocrit and reticulocytes are plotted (left). White blood cell (WBC) and absolute neutrophil counts (ANC) are plotted (right). Green arrow on day 0 indicates the start date of itacitinib. d. Bone marrow hematoxylin and eosin (H\&E) and phosphorylated STAT1 (pSTAT1) staining are shown for a healthy donor (left) and the patient (right). Sections from the patient pre-itacitinib and post-itacitinib at two separate time-points are shown.

a.

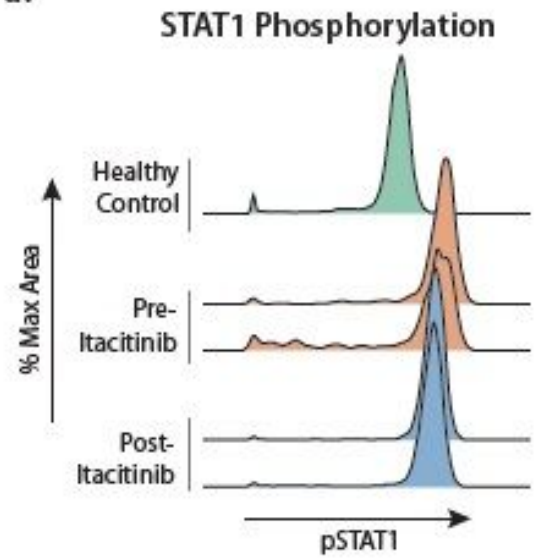

c.
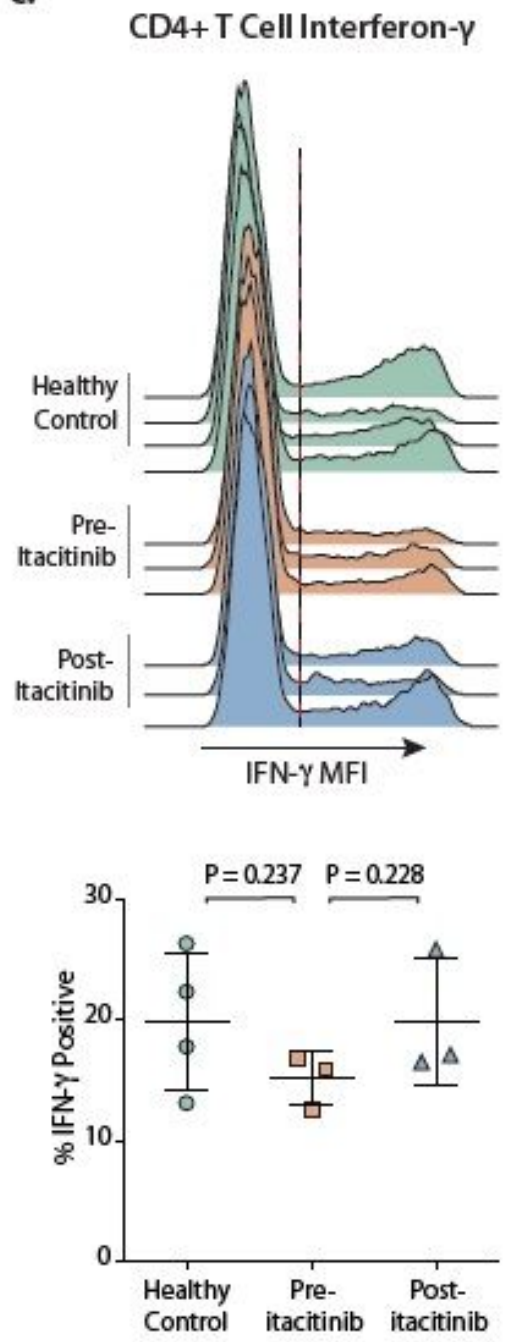

b.
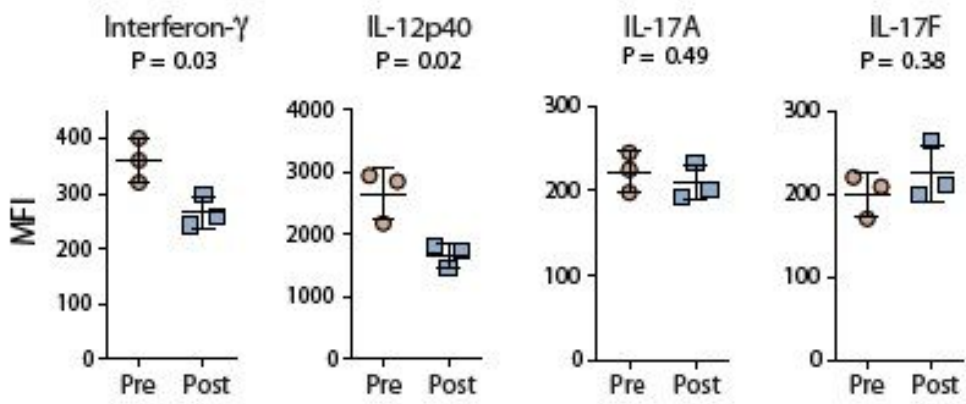

d.

CD8+ T Cell Interferon- $\gamma$
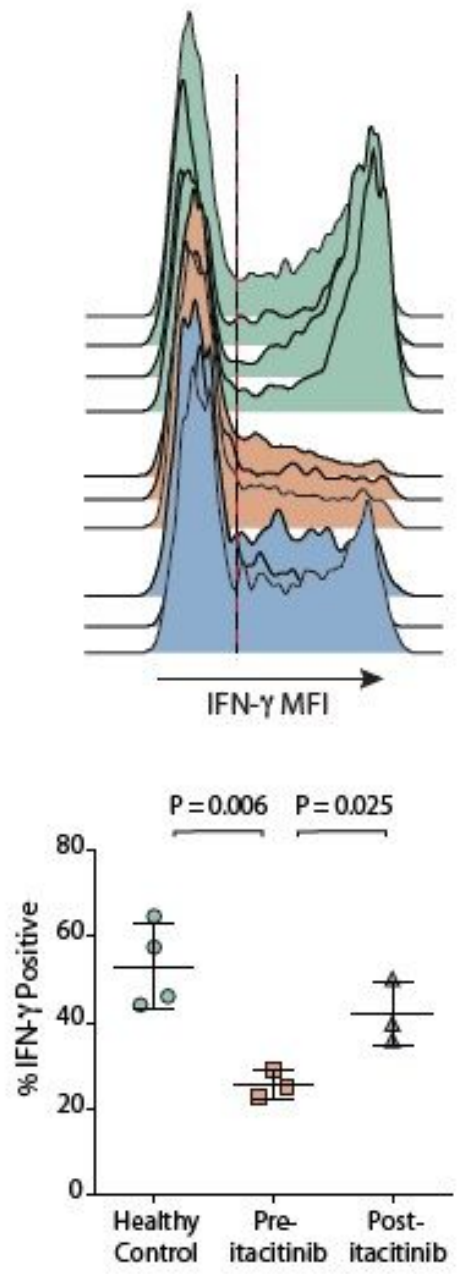

e.

\section{CD8+T Cell PD-1}
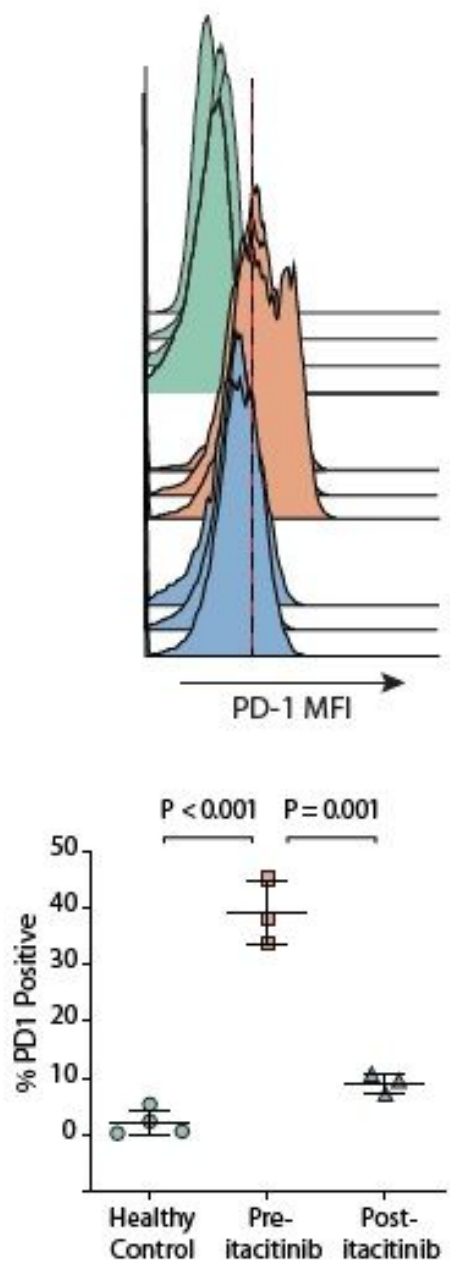

Figure 2 
Immunophenotyping. a. Phospho-CyTOF histograms pSTAT1 levels after stimulation in vitro with interferon-y. Shown are pSTAT1 levels in monocytes from a healthy control and four different time points from the patient pre- and post-itacitinib treatment. b. Plasma cytokine levels measured in units of mean fluorescent intensity (MFI) from the patient at six time points pre- and post-itacitinib. Each sample was measured in duplicate. c. CD4+ memory T cells (CD45RO+) are shown both as histograms and the gated percent of cells secreting IFN-y. d. CD8+ memory T cells (CD45RO+) are shown both as histograms and the gated percent of cells secreting IFN-y. e. Programmed Death 1 (PD-1) expression levels on CD8+ T cells are shown as histograms and as the gated percent of cells expressing PD-1. (c.- d.) PBMCs were stimulated in vitro with PMA/ionomycin and stained for intracellular cytokines. (c.- e.) Healthy control $n=4$, pre-itacitinib $n=3$, post-itacitinib $n=3$. (b.- e.) Statistical analyses performed using unpaired t-test.

a.
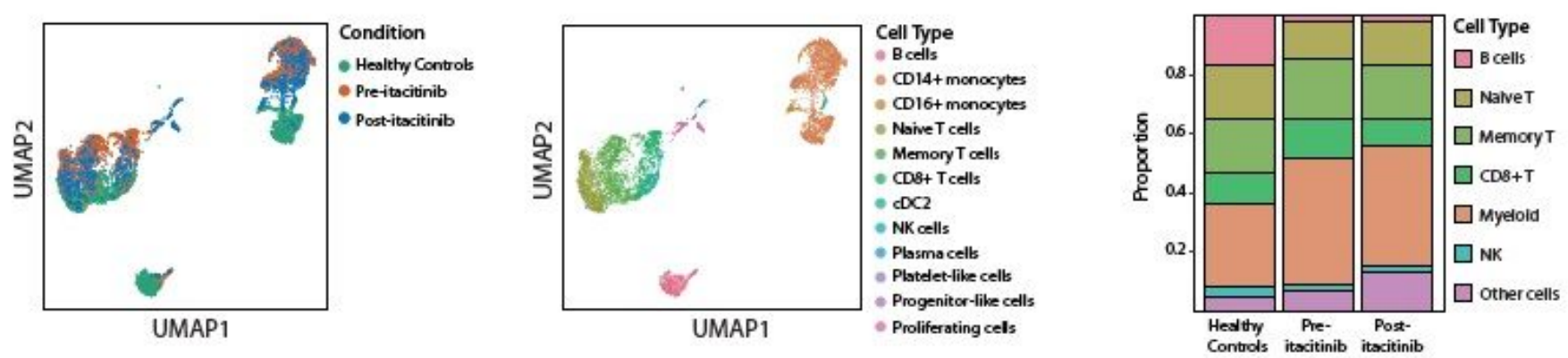

b.

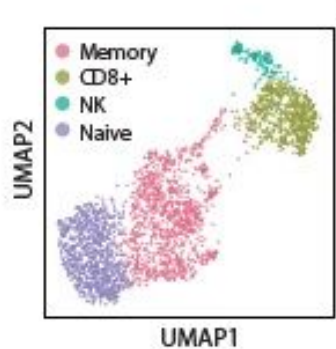

TCells
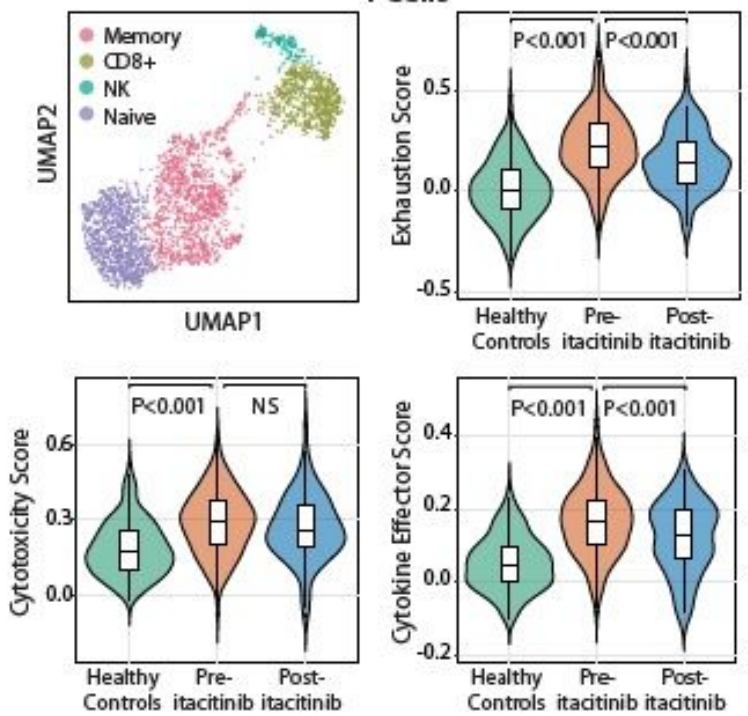

d.

scATAC-Seq PD-1 Locus

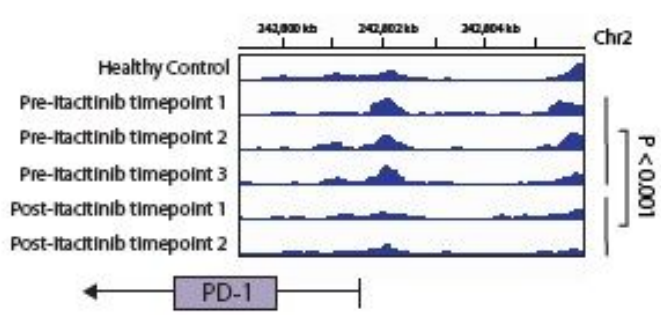

c.
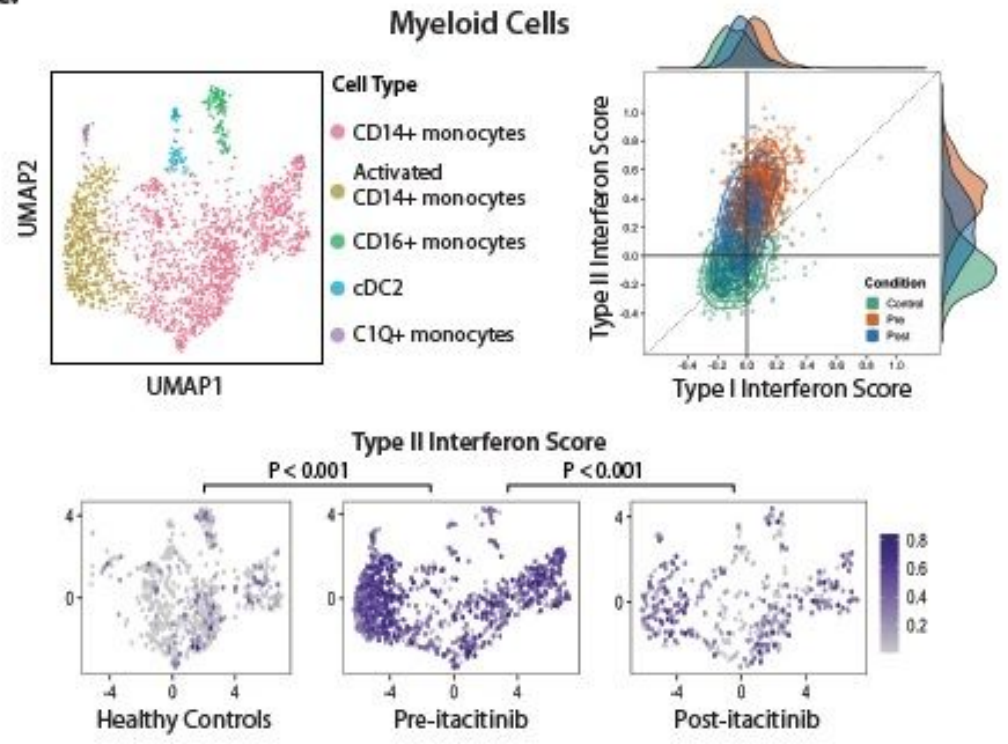

Type II Interferon Score

$P<0.001$

e.

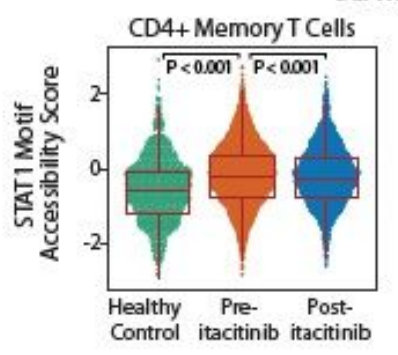

scATAC-Seq STAT1 Accessibility
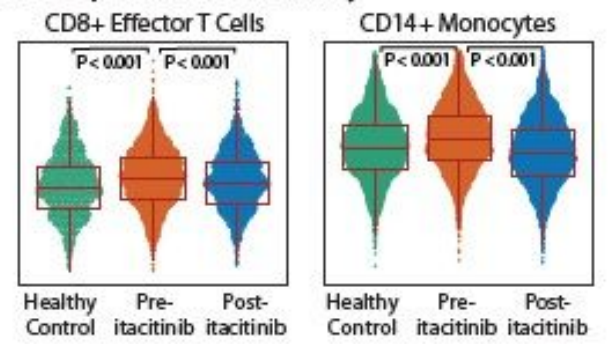
Single-cell transcriptional and epigenetic profiling. a. Unsupervised clustering of all cells by scRNA-Seq expression profiling in Uniform Manifold Approximation and Projection (UMAP) space. Cells are colored by condition (left) or cell type (middle). $n=4$ different healthy control samples, $n=3$ pre-itacitinib time points, and $n=3$ post- itacitinib time points are included. Stacked bar plot shows cell proportions by condition (right). "Myeloid" includes CD14+ monocytes, CD16+ monocytes, and type 2 conventional dendritic cells (CDC2). "Other" cells include plasma cells, platelet-like cells, progenitor-like cells, and proliferating cells. b. Sub-clustering of T cell-containing populations (top left). The CD8+ T cell population (olive green) was then scored for exhaustion, cytotoxicity, and cytokine effector function and plotted by condition. c. Sub-clustering of myeloid populations (top left). Each myeloid cell was scored and plotted (top right) by its Type I interferon score (x-axis) and Type II interferon score (y-axis). Histograms of each of these scores by condition are shown on the plot edge. Each myeloid cell was then plotted by condition and colored by its Type II interferon score (bottom figures). d. scATAC-seq accessibility peaks at the PD-1 locus in healthy control and the patient's PBMCs at five time points. e. scATAC-seq genome-wide accessibility at STAT1 binding motif sites. Across cell types and conditions as labeled, each cell was scored by STAT1 accessibility. P values calculated using Wilcoxon rank-sum test.

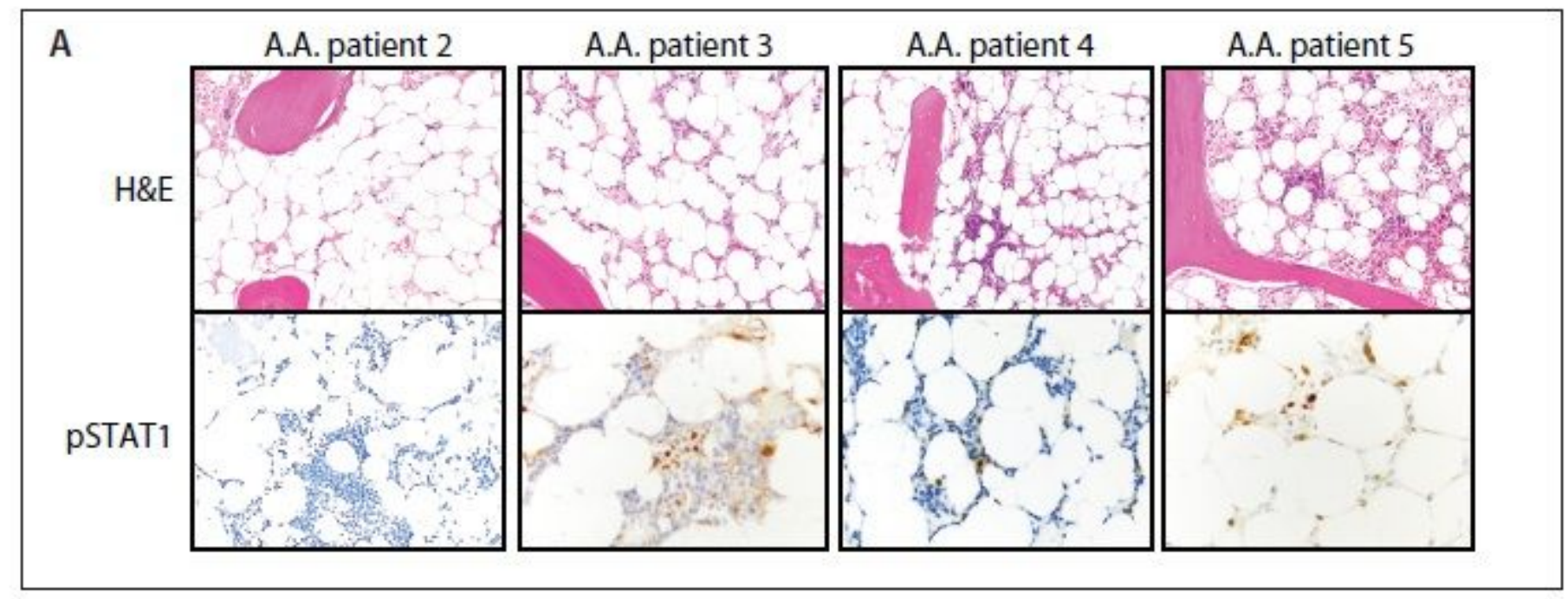

\section{Figure 4}

Bone marrow STAT1 activation in historical aplastic anemia cases. Bone marrow hematoxylin and eosin (H\&E) and phosphorylated STAT1 (pSTAT1) staining are shown for four historical patients with idiopathic aplastic anemia (A.A.) at the time of their diagnosis.

\section{Supplementary Files}

This is a list of supplementary files associated with this preprint. Click to download.

- STAT1Methods.pdf

- TableS1.xlsx 
- SupplementalFigures.pdf 